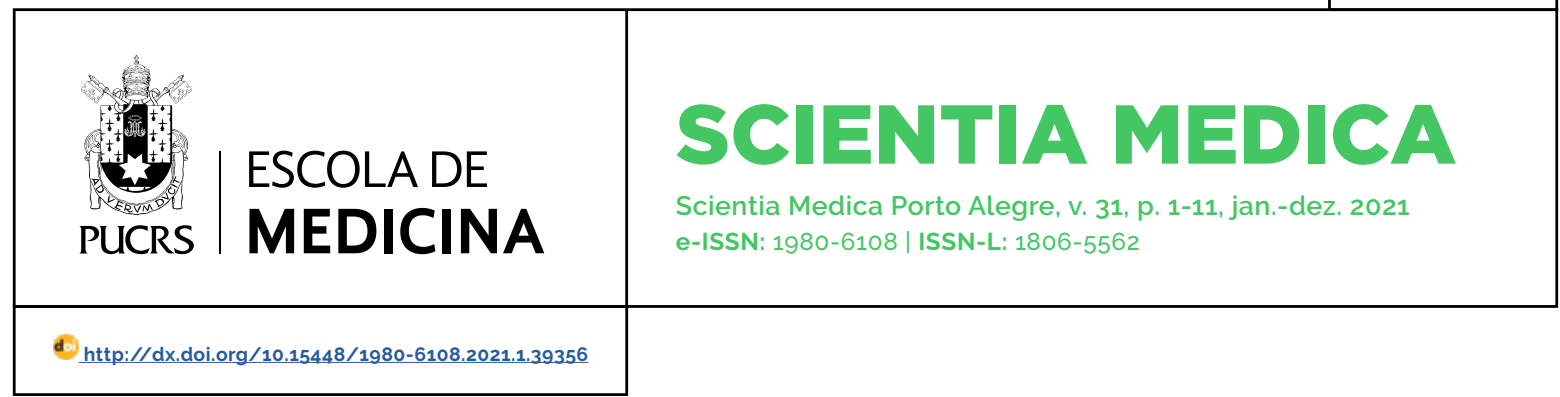

ARTIGO ORIGINAL

\title{
Intervenções de fisioterapia respiratória utilizadas durante a hospitalização de crianças e adolescentes com asma: relatos profissionais
}

\author{
Respiratory physiotherapy interventions used during hospitalization of children and \\ adolescents with asthma: professional reports
}

\section{Daniele Oppermann Ruckert $^{1}$}

orcid.org/0000-0002-4060-4381 daniele_oppermannahotmail.com

\section{Márcio Vinícius Fagundes Donadio 2} orcid.org/0000-0001-8836-9109 mdonadio@pucrs.br

\section{João Paulo Heinzmann- Filho $^{1}$}

orcid.org/0000-0002-8426-0250 joaopauloheinzmann@hotmail.com

Recebido em:14 out. 2020 Aprovado em: 29 maio 2021 Publicado em: 8 jul. 2021

\section{(c) (i)}

Artigo está licenciado sob forma de uma licença Creative Commons Atribuição 4.0 Internacional.

\section{Resumo}

Objetivo: descrever e comparar as intervenções de fisioterapia respiratória utilizadas para asma durante a hospitalização em três grupos etários pediátricos. Além disso, buscou-se investigar os motivos de escolha dessas intervenções.

Métodos: a amostra foi composta por fisioterapeutas atuantes em hospitais que reportaram atender crianças e adolescentes com asma. Os profissionais responderam a um questionário online sobre dados pessoais, acadêmicos, profissionais e relativo às intervenções de fisioterapia respiratória utilizadas em lactentes, pré-escolares e escolares/adolescentes. As intervenções foram agrupadas em nove classificações: convencionais, manuais, baseadas em volume, oscilação oral de alta frequência/pressão expiratória positiva (OOAF/PEP), exercicios ventilatórios, ventilação não invasiva, técnica de expiração forçada (TEF), aspiração de vias aéreas superiores (VAS) e outras.

Resultados: foram incluídos 93 fisioterapeutas, com idade entre 31 e 40 anos $(47,3 \%)$ e do sexo feminino $(87,1 \%)$. As intervenções mais utilizadas nos lactentes foram a aspiração de VAS (78.5\%), a aceleração do fluxo expiratório (AFE) (50.5\%) e a terapia expiratória manual passiva (TEMP) (45,2\%). Nos pré-escolares, predominou a tosse (75.3\%), a aspiração de VAS (52,7\%), a AFE (51,6\%) e a TEMP/expiração lenta e prolongada (ELPr) (50,5\%). Já nos escolares/adolescentes, a tosse (83.9\%), os exercícios expiratórios variados (73,1\%) e a ELPr $(57,0 \%)$ sobressairam-se. Houve menor utilização $(p<0,01)$ de OOAF/PEP, de exercícios ventilatórios e de TEF nos lactentes e, também, de métodos convencionais, manuais, aspiração de VAS e outras terapias $(p<0,01)$ nos escolares/adolescentes. Os profissionais relataram utilizar essas intervenções por serem mais eficazes na prática clínica (78,5\%).

Conclusão: as intervenções manuais e as técnicas de expectoração visando à desobstrução brônquica foram as mais frequentemente utilizadas, tendo relação com a faixa etária e a escolha devido à efetividade na prática clínica.

Palavras-chave: asma, modalidades de fisioterapia, terapia respiratória, hospitalização, pediatria.

\section{Abstract}

Aims: to describe and to compare the airway clearance techniques used for asthma during hospitalization in three pediatric age groups. In addition, we sought to investigate the main reasons for choosing the interventions.

Methods: the sample consisted of physiotherapists working in hospitals who reported attending children and adolescents with asthma. The professionals answered an online questionnaire on personal, academic and professional data, as well as regarding the airway clearance techniques used in, preschoolers and schoolchildren/adolescents. The interventions were grouped into nine classifications: conventional, manual, volume-based, high-frequency oral oscillation/ positive expiratory pressure (HFOO/PEP), ventilatory exercises, non-invasive 
ventilation, forced expiratory technique (FET), upper airway aspiration (UAA) and others.

Results: nine-three physiotherapists were included, aged between 31 and 40 years (47.3\%) and female (87.1\%). The most frequent interventions in infants were UAA (78.5\%), expiratory flow acceleration (EFA) (50.5\%) and chest compression (45.2\%). In preschoolers, coughing $(75.3 \%)$, aspiration of upper airways (52.7\%), EFA (51.6\%) and chest compression/slow and prolonged expiration (SPE) (50.5\%) were the most used. In schoolchildren/ adolescents, coughing (83.9\%), varying expiratory exercises $(73.1 \%)$ and SPE (57.0\%) were the most frequent. There was less use $(p<0.01)$ of OOAF/PEP, ventilatory and FET exercises in infants and also of conventional, manual methods, aspiration of UUA and other therapies $(p<0.01)$ in schoolchildren/adolescents. The professionals reported using these interventions because they are more effective in clinical practice $(78.5 \%)$.

Conclusions: manual and expectoration techniques aiming at airway clearance were the most frequently used, being related to the age group and chosen due to effectiveness in clinical practice.

Keywords: asthma, physiotherapy modalities, respiratory therapy, hospitalization, pediatrics.

ABREVIATURAS: AFE, aceleração de fluxo expiratório; BIPAP, Bi-level Positive Airway Pressure; ELPr, expiração lenta e prolongada; FR; fisioterapia respiratória; PEP, pressão expiratória positiva; RTA, reequilibrio toracoabdominal; TEMP, terapia expiratória manual passiva; VAS, vias aéreas superiores.

\section{Introdução}

A asma é considerada uma doença inflamatória crônica, que resulta em restrição variável do fluxo aéreo, podendo ser reversivel espontaneamente ou com tratamento. Manifesta-se por episódios de sibilos, dispneia, opressão torácica e tosse, especialmente, à noite e pela manhã, ao despertar (1).

É considerada a doença crônica mais comum na infância, gerando custos indiretos e diretos para a sociedade (2). No mundo, estimam-se 300 milhões de indivíduos com asma, obtendo uma prevalência global de 10\% (3, 4). No Brasil, o número de acometidos já passa de 20 milhões, com prevalência de 20\% em jovens na região Sul do país (5). Anualmente, ocorrem 350 mil hospitalizações por asma no Brasil, constituindo-se a quarta causa de hospitalizações pelo Sistema Único de Saúde e o terceiro motivo entre crianças e adultos jovens (6).

Essas hospitalizações, frequentemente, são causadas por períodos de crise, podendo ser oriundas de diversas substâncias e fatores alérgenos, bem como processos infecciosos (1). Fisiologicamente, isso desencadeia a hiperresponsividade e a obstrução brônquica, além da ocorrência de edema e do acúmulo de muco no sistema pulmonar, comprometendo a impedância respiratória $(1,7)$. O tratamento da asma engloba diferentes mecanismos que visam manter o controle da doença (8), incluindo agentes farmacológicos, como os beta-2 agonistas inalatórios de curta duração, glicocorticoides orais e os anticolinérgicos inalatórios. Além disso, os antibióticos podem ser utilizados no processo de hospitalização (7).

Na maioria dos hospitais, a fisioterapia respiratória (FR) é vista como parte integrante do tratamento de crianças e de adolescentes com asma, apesar das evidências científicas serem ainda incipientes quanto a sua efetividade (9-11). Dentre os efeitos positivos da FR, podem-se listar a redução da sintomatologia e o controle da doença, a melhora do sincronismo tóraco-abdominal e os ganhos no desempenho cardiorrespiratório (12). Todavia, outros achados apontam ausência de efeito clínico, limitações metodológicas dos estudos, além das variáveis limitadoras resultantes da própria hospitalização. Esses aspectos dificultam a elaboração de condutas clínicas para pacientes com asma hospitalizados $(13,14)$.

Neste sentido, a recomendação da fisioterapia acaba tendo como base os aspectos fisiopatológicos envolvidos na doença e a melhora clínica observada na prática profissional. A FR tem como principais objetivos desobstruir as vias aéreas, melhorar a relação ventilação-perfusão e otimizar a impedância pulmonar comumente afetada pela doença (15). Para isso, conta com diversas intervenções, incluindo as terapias convencionais e manuais, baseadas em volume, dispositivos de oscilação oral de alta frequência/pressão expiratória positiva (OOAF/PEP), exercícios ventilatórios, entre outras (16). A escolha delas está comumente relacionada à idade, à preferência do paciente e à experiência profissional, já que as evidências científicas ainda são incipientes (17).

Portanto, considerando que as intervenções fisioterapêuticas são utilizadas em unidades de internação e que até o presente momento os achados disponiveis na literatura são divergentes quanto a sua efetividade $(10,17)$, é necessário um maior conhecimento em relação às condutas 
terapêuticas mais utilizadas pelos profissionais. Assim, o objetivo do estudo foi descrever as intervenções de FR utilizadas durante a hospitalização em crianças e adolescentes com asma. Secundariamente, buscou-se comparar a frequência de realização entre três diferentes faixas etárias, além de investigar os motivos de escolha das intervenções.

\section{Métodos}

Trata-se de um estudo transversal, realizado utilizando-se um questionário online. O questionário foi preenchido por fisioterapeutas, atuantes em hospitais localizados no Rio Grande do Sul (RS), e que reportaram atender crianças e adolescentes (até 18 anos) com asma. Além disso, era necessário aceitar participar do estudo através da leitura e aceite do Termo de Consentimento Livre e Esclarecido (TCLE). Por outro lado, os profissionais que responderam de forma incompleta o instrumento, além daqueles que duplicaram o envio das respostas, foram excluidos.

O link de acesso ao questionário e ao TCLE foi enviado por e-mail para os profissionais, via Conselho Regional de Fisioterapia e Terapia Ocupacional da 5. ${ }^{\text {a }}$ região (CREFITO-5) e divulgado nas redes sociais pelos pesquisadores do estudo. O período de registro das respostas ocorreu entre os meses de abril e maio de 2020.

O questionário utilizado foi criado pelos pesquisadores, sendo composto por 15 questões. As perguntas incluiram as seguintes informações: idade, sexo, tempo de formação, tempo de atuação em hospital, nivel de formação, aperfeiçoamento (pós-graduação stricto sensu ou lato sensu) em pediatria, gravidade da asma, tempo de atendimento, terapia inalatória associada e as intervenções de FR utilizadas.

Foram disponibilizadas 24 diferentes intervenções de FR no questionário, incluindo: drenagem postural, vibração/vibrocompressão, tapotagem, terapia expiratória manual passiva (TEMP), aceleração de fluxo expiratório (AFE), expiração lenta e prolongada (ELPr), desobstrução rinofaringea retrograda (DRR), expiração lenta total com a glote aberta (ELTGOL), manobras expansivas, drena- gem autogênica (DA), ciclo ativo da respiração (CAR), máscara de PEP, selo d'água, Flutter ${ }^{\circledR} /$ Shaker $₫ /$ Acapella $\AA$, Respiron $\AA /$ voldayne $\AA$, respiração diafragmática, suspiros/soluços inspiratórios, exercícios respiratórios com expiração prolongada, Bi-level Positive Airway Pressure (BIPAP)/Continuous Positive Airway Pressure (CPAP), tosse, huffing, aspiração de vias aéreas superiores (VAS), reequilibrio toracoabdominal (RTA) e treinamento muscular respiratório (TMR).

Posteriormente, essas intervenções foram agrupadas em nove outras classificações, de forma adaptada (18), sendo essas: a) Convencionais: drenagem postural, vibração/vibrocompressão, tapotagem; b) Manuais: TEMP, AFE, ELPr, DRR, ELTGOL e manobras expansivas; c) Baseadas em volume: DA e CAR; d) OOAF/PEP: máscara de PEP, selo d'agua e Flutter $\AA /$ Shaker $\AA /$ Acapella $($; e) Exercícios ventilatórios: respiron $\AA /$ voldayne $($, respiração diafragmática, suspiros/soluços inspiratórios, exercícios respiratórios com expiração prolongada; f) Ventilação não invasiva (VNI): BIPAP/ CPAP; g) Técnica de expiração forçada (TEF): tosse e huffing; h) Aspiração de VAS; e i) Outras: RTA e TMR.

O presente estudo foi aprovado pelo Comitê de Ética em Pesquisa (CEP) do Centro Universitário Cenecista de Osório (UNICNEC), sob o n. ${ }^{\circ}$ 4.008.940, e obedece a resolução do Conselho Nacional de Saúde (CNS) por meio das resoluções n. 196/1996 e n. $446 / 2012$. Todos os participantes envolvidos aceitaram participar da pesquisa por meio do TCLE.

Para a análise estatística, os dados qualitativos foram apresentados em frequência absoluta (n) e relativa (\%). A análise das diferentes classificações de FR entre as três faixas etárias (lactentes: <2 anos; pré-escolares: 2 até 6 anos; escolares/adolescentes: 7 até 18 anos) (19) foi investigada pelo teste de qui-quadrado de Pearson, com análise de resíduos ajustados. O valor da significância estatística foi de $5 \%$. Todas as análises foram realizadas com o programa PASW Statistics 17.

\section{Resultados}

De um total de 150 acessos ao link enviado aos profissionais, 148 (98,7\%) aceitaram participar do estudo. Desses, 146 (97.3\%) responderam 
completamente o questionário, sendo que 53 (36.3\%) foram excluidos por não preencherem os critérios de elegibilidade. Assim, a amostra foi composta de 93 fisioterapeutas que relataram atender crianças e adolescentes com asma durante a hospitalização.

A maioria dos profissionais indicou ter idade entre 31 e 40 anos e ser do sexo feminino. Mais da metade deles informaram possuir especialização/ residência (Tabela 1). Além disso, os fisioterapeutas reportaram atender com mais frequência asma de gravidade moderada/grave (Tabela 2).

TABELA 1 - Características dos fisioterapeutas participantes do estudo.

\begin{tabular}{ccc}
\hline Variáveis & $\mathbf{n}$ & $\%$ \\
\hline Idade (anos) & & \\
$20-30$ & 40 & 43,0 \\
$31-40$ & 44 & 47,3 \\
$41-50$ & 08 & 8,6 \\
$51-60$ & - & - \\
$\geq 61$ & 01 & 1,1
\end{tabular}

Sexo, $n(\%)$

$\begin{array}{lll}\text { Feminino } & 81 & 87,1 \\ \text { Masculino } & 12 & 12,9\end{array}$

Tempo de formação, anos

$\begin{array}{lll}\leq 5 & 47 & 50,5 \\ 6-10 & 17 & 18,3 \\ 11-15 & 17 & 18,3 \\ 16-20 & 06 & 6,5 \\ \geq 21 & 06 & 6,5\end{array}$

Tempo de atuação hospitalar, anos

$\leq 5$

$6-10$

$11-15$

$16-20$

$\geq 21$

$\begin{array}{ll}52 & 55,9 \\ 21 & 22,6 \\ 11 & 11,8 \\ 06 & 6,5 \\ 03 & 3,2\end{array}$

Formação concluida

$\begin{array}{lcc}\text { Graduação } & 14 & 15,1 \\ \text { Especialização/residência } & 60 & 64,5 \\ \text { Mestrado } & 14 & 15,1 \\ \text { Doutorado } & 05 & 5,4\end{array}$

Aperfeiçoamento em pediatria concluido*

\begin{tabular}{lll} 
Sim & 51 & 54,8 \\
Não & 42 & 45,2 \\
\hline
\end{tabular}

"Aperfeiçoamento em pediatria (pós-graduação stricto sensu ou lato sensu).
TABELA 2 - Gravidade, terapia inalatória e tempo gasto no atendimento de pacientes asmáticos durante a hospitalização.

\begin{tabular}{lcc}
\hline Variáveis & $\mathbf{n}$ & $\%$ \\
\hline Gravidade da asma & 25 & 26,9 \\
$\quad$ Leve & 68 & 73,1 \\
$\quad$ Moderada a grave & & \\
Terapia inalatória associada & 74 & 79,6 \\
$\quad$ Sim & 19 & 20,4 \\
$\quad$ Não & & \\
Momento da terapia inalatória & 66 & 71,0 \\
$\quad$ Antes da fisioterapia & 16 & 17,2 \\
$\quad$ Depois da fisioterapia & 11 & 11,8 \\
$\quad$ Não se aplica & & \\
Atendimento fisioterapêutico, minutos & & \\
5 - 10 & 07 & 7,5 \\
11 - 20 & 52 & 55,9 \\
21 - 30 & 29 & 31,2 \\
\hline A 31 & 05 & 5,4 \\
\hline
\end{tabular}

As intervenções de FR mais utilizadas nos lactentes foram o procedimento de aspiração de VAS, seguido de técnicas manuais, além de outras terapias. Nos pré-escolares, predominou a tosse, acompanhado pelo procedimento de aspiração de VAS e, novamente, de intervenções manuais. Já nos escolares/adolescentes, a tosse, os exercícios expiratórios variados e as intervenções manuais foram as mais utilizadas (Tabela 3). 
TABELA 3 - Descrição das diferentes intervenções de fisioterapia respiratória relatadas pelos profissionais de acordo com três grupos etários.

Intervenções fisioterapêuticas $\quad$ Lactentes $\quad$ Pré-escolares $\begin{gathered}\text { Escolares - } \\ \text { Adolescentes }\end{gathered}$

\begin{tabular}{|c|c|c|c|c|c|c|}
\hline Convencionais & $\mathbf{n}$ & $\%$ & n & $\%$ & n & $\%$ \\
\hline Drenagem postural & 21 & 22,6 & 22 & 23.7 & 13 & 14,0 \\
\hline Tapotagem & - & - & - & - & - & - \\
\hline Vibração/vibrocompressão & 31 & 33.3 & 38 & 40,9 & 22 & 23,7 \\
\hline \multicolumn{7}{|l|}{ Manuais } \\
\hline Técnica de expiração manual passiva & 42 & 45,2 & 47 & 50,5 & 43 & 46,2 \\
\hline Aceleração de fluxo expiratório & 47 & 50,5 & 48 & 51,6 & 35 & 37,6 \\
\hline Desobstrução rinofaringea retrógrada & 25 & 26,9 & 10 & 10,8 & 03 & 3,2 \\
\hline Expiração lenta e prolongada & 35 & 37,6 & 47 & 50,5 & 53 & 57,0 \\
\hline Expiração lenta com a glote aberta & 07 & 7.5 & 07 & 7,5 & 09 & 9.7 \\
\hline Manobras expansivas & 28 & 30,1 & 32 & 34,4 & 33 & 35.5 \\
\hline \multicolumn{7}{|l|}{ Baseadas em volume } \\
\hline Drenagem autogênica & 05 & 5.4 & 02 & 2,2 & 08 & 8,6 \\
\hline Ciclo ativo da respiração & $\mathrm{O} 2$ & 2,2 & 10 & 10,8 & 31 & 33.3 \\
\hline \multicolumn{7}{|l|}{ OOAF/PEP } \\
\hline Flutter ${ }^{\circledR}$, Shaker ${ }^{\circledR}$ ou Acapella ${ }^{\circledR}$ & 06 & 6,5 & 28 & 30,1 & 43 & 46,2 \\
\hline Máscara de pressão expiratória positiva & ०8 & 8,6 & 17 & 18,3 & 22 & 23.7 \\
\hline Selo d'água & 06 & 6,5 & 37 & 39,8 & 45 & 48,4 \\
\hline
\end{tabular}

Exercícios ventilatórios

$\begin{array}{lllllll}\text { Respiron } ® \text { ou Voldayne }{ }^{\circledR} & 01 & 1,1 & 06 & 6,5 & 13 & 14,0 \\ \text { Respiração diafragmática } & 10 & 10,8 & 24 & 25,8 & 45 & 48,4 \\ \text { Suspiros/soluços inspiratórios } & 01 & 1,1 & 04 & 4,3 & 17 & 18,3 \\ \text { Exercícios expiratórios variados } & 14 & 15,1 & 43 & 46,2 & 68 & 73,1 \\ \text { ntilação não invasiva } & & & & & & 38,7 \\ \text { BIPAP ou CPAP } & 38 & 40,9 & 35 & 37,6 & 36 & 38\end{array}$

Técnicas de expiração forçada

$\begin{array}{lllllll}\text { Tosse } & 40 & 43,0 & 70 & 75,3 & 78 & 83.9 \\ \text { Huffing } & 10 & 10,8 & 37 & 39,8 & 52 & 55.9\end{array}$

Procedimento invasivo

$\begin{array}{lllllll}\text { Aspiração de vias aérea superiores } & 73 & 78,5 & 49 & 52,7 & 22 & 23.7\end{array}$

Outras

$\begin{array}{lllllll}\text { Reequilibrio tóraco-abdominal } & 40 & 43,0 & 33 & 35.5 & 22 & 23.7 \\ \text { Treinamento muscular respiratório } & - & - & - & - & - & -\end{array}$

BIPAP, Bi-level Positive Airway Pressure; CPAP: Continuous Positive Airway Pressure; OOAF, Oscilação oral de alta frequência; PEP, Pressão expiratória positiva. 


\section{6/11 Scientia Medica Porto Alegre, v. 31, p. 1-11, jan.-dez. 2021 | e-39356}

Houve menos preferência por OOAF/PEP, exercícios ventilatórios e de TEF nos lactentes, bem como baixo uso de intervenções convencionais, manuais, do procedimento de aspiração de VAS e de outras terapias nos escolares/adolescentes.

As intervenções baseadas em volume foram mais prevalentes nesse último grupo (Figura 1).
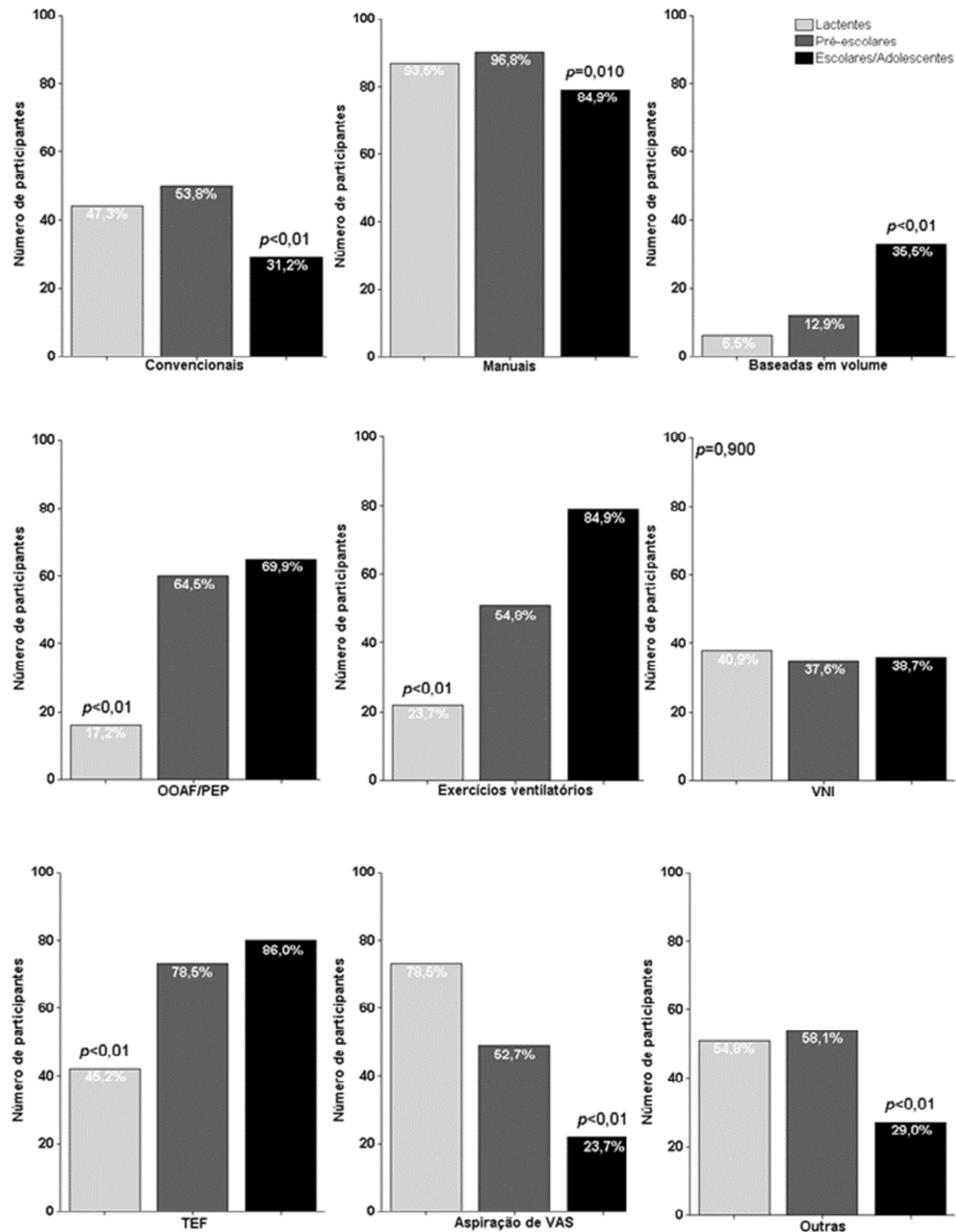

Figura 1 - Comparação de diferentes classificações das intervenções fisioterapêuticas relatadas pelos profissionais entre três faixas etárias avaliadas. 
A maioria dos profissionais relatou que utilizou essas intervenções por verificar que são mais eficazes na prática clínica (Figura 2).

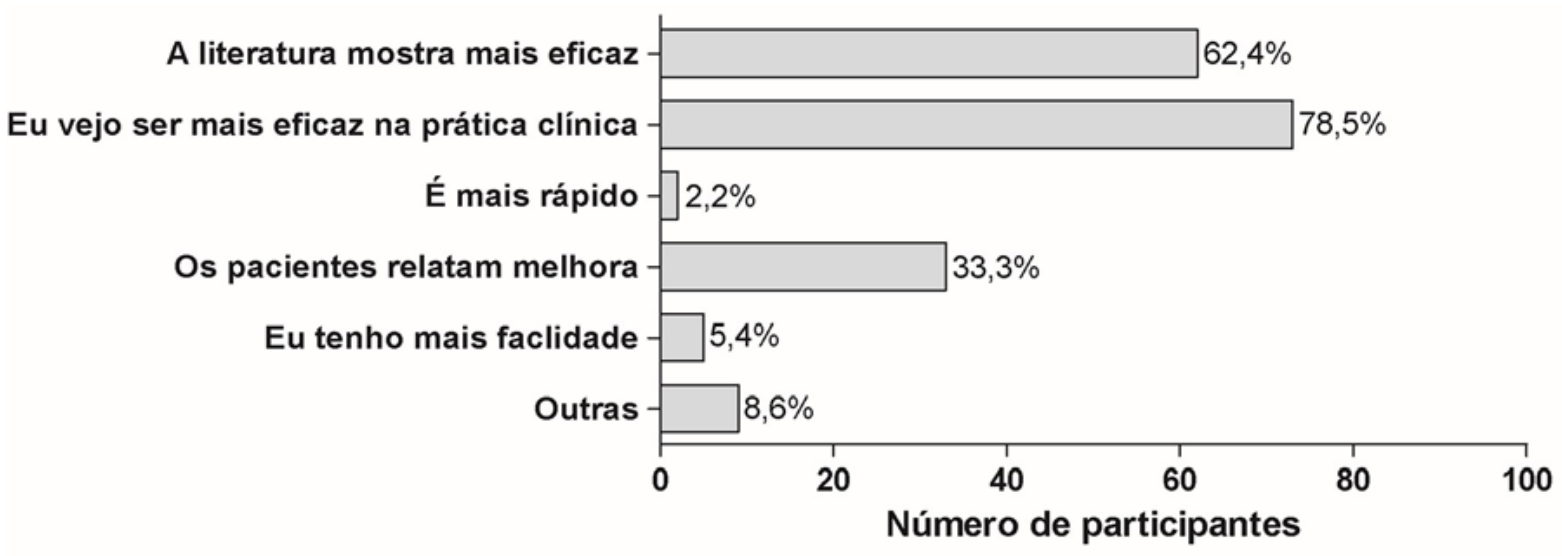

Figura 2 - Motivos de escolha das intervenções de fisioterapia respiratória para pacientes com asma hospitalizados.

\section{Discussão}

As intervenções de FR são amplamente utilizadas pelos profissionais no contexto hospitalar, com diversas finalidades, de acordo com a indicação terapêutica. Primordialmente, grande parte das intervenções possui o objetivo de melhorar a obstrução brônquica, considerando a presença de secreção pulmonar típica nestes pacientes durante a hospitalização (20). Além disso, algumas intervenções têm o intuito de gerar expansão e/ou desinsuflação pulmonar, dependendo da condição clínica $(20,21)$. A partir de nosso conhecimento, este é o primeiro estudo que realizou um levantamento das principais intervenções fisioterapêuticas utilizadas pelos profissionais durante a hospitalização de crianças e de adolescentes com asma.

Os achados deste trabalho demonstraram que o procedimento aspiração de VAS e a TEF foram as intervenções de fisioterapia mais utilizadas pelos profissionais nesta doença. Como esperado, independentemente do tipo de intervenção, os atendimentos hospitalares de fisioterapia costumam finalizar com a aspiração de VAS, huffing e/ ou tosse. A escolha pela aspiração de VAS ocorre na presença de tosse produtiva e/ou ineficaz, além de ser mais executada nos pacientes mais jovens (22), fato essa maior utilização nos lacten- tes. Da mesma forma, a execução do huffing e/ou da tosse (voluntária ou provocada) é uma forma não invasiva para executar e finalizar a "limpeza" de vias aéreas (22). O intuito do reflexo da tosse é promover o aumento do fluxo expiratório para causar a depuração das secreções pulmonares (23). Corroborando com nossos achados, um estudo multicêntrico realizado em pacientes com fibrose cística evidenciou que o huffing foi a intervenção mais recomendada pelos fisioterapeutas em centros de referência (18). Embora elas sejam doenças crônicas de origens diferentes, ambas possuem comprometimento ventilatório obstrutivo, o que poderia, pelo menos em parte, explicar a similaridade nos resultados.

As intervenções manuais, tais como a TEMP, AFE e a ELPr, foram as mais utilizadas nos lactentes e pré-escolares. Além disso, foi a segunda classificação mais indicada nos escolares e adolescentes. A TEMP e a ELPr se baseiam na geração passiva de fluxo expiratório lento e/ou prolongado, obtendo na ELPr também um apoio abdominal (24). Estas técnicas têm como princípio fisiológico a desobstrução de vias aéreas médias/ periféricas. Nogueira et al. (24) demonstrou uma excelente reprodutibilidade de execução da ELPr entre três fisioterapeutas em uma amostra de lactentes com sibilos, indicando ser uma mano- 
bra de fácil aplicabilidade clínica. Já a AFE, tem como intuito gerar fluxo expiratório rápido, de forma passiva, atuando na desobstrução de vias aéreas centrais (25). Outro fato interessante foi o registro da aplicação do RTA nos lactentes pelos participantes do estudo. Essa intervenção objetiva normalizar o tônus através de um conjunto de manuseios dinâmicos sobre o tronco, motivo esse, que nos levou a classificar essa intervenção como outras terapias. No entanto, não existe registro na literatura que suporte essa recomendação (21).

A escolha das intervenções de FR está comumente relacionada à idade, à preferência do paciente, ao grau de independência da terapia, à experiência profissional e a evidências científicas (17). No presente estudo, houve menores relatos de intervenções baseadas em OOAF/PEP, exercicios ventilatórios e de TEF nos lactentes e, também, menor frequência de intervenções convencionais, manuais, do procedimento de aspiração de VAS e outras terapias (RTA) nos escolares/adolescentes. Isso pode ser atribuído às diferentes independências dos pacientes nestes grupos etários, considerando que em idades mais jovens seria inviável o uso de intervenções volitivas. Sabe-se também que intervenções mais independentes devem ser a escolha, preferencialmente, para amostras pediátricas com mais idade, já que proporcionam maior envolvimento e adesão na terapia (20, 21). Lanza et al. (26), reportaram em uma amostra de pacientes com pneumonia, que o uso da OOAF/ TEF tem melhor aplicabilidade em escolares e adolescentes, visto que esses compreendem com maior facilidade a intervenção, além de gerar menor gasto energético quando comparado a outras técnicas. Além disso, as intervenções baseadas em volume, como a DA e o CAR, foram mais utilizadas nos escolares e nos adolescentes, já que se pressupõe maior entendimento e independência (27). A sua utilização pode ser uma alternativa para a remoção de secreção das vias aéreas em amostras pediátricas mais independentes, já que esses pacientes permanecem hospitalizados por cerca de 10 dias (28).

Na prática clínica, o uso da inaloterapia é um recurso utilizado frequentemente antes da FR com objetivo de broncodilatação da via aérea e facilitação da remoção de secreções (29). A utilização de broncodilatador de curta duração serve para reverter a obstrução do fluxo aéreo, podendo ser administrado através da nebulização ou de inalador dosimetrado (29, 30). Isto corrobora com nossos resultados, já que a maior parte dos profissionais relatou associar a terapia inalatória prescrita com a FR, sendo executada a priori do atendimento. Historicamente, a terapia mais utilizada na administração de broncodilatador é a nebulização, por ser comumente utilizada na rotina hospitalar e, também, pelo baixo custo (29). No entanto, uma metanálise recente encontrou que tanto o inalador dosimetrado como a nebulização são igualmente eficazes na exacerbação da asma em crianças (30).

O principal motivo relatado pelos profissionais na escolha das intervenções de FR foi "Eu vejo ser eficaz na prática clínica", sugerindo uma limitada aplicação da prática baseada em evidências. Isso pode ser reflexo dos poucos estudos sobre o tema e pelas limitações dos trabalhos no ambiente hospitalar $(9,10)$. Estes achados vão de encontro a outro estudo que descreveu esse mesmo motivo como o mais assinalado (38,3\%) por profissionais em Unidade de Terapia Intensiva (31). Paradoxalmente, embora algumas revisões tenham reportado não haver evidências científicas que suportem a indicação dessas intervenções no tratamento da asma $(13,14)$, o segundo motivo mais indicado foi "a literatura mostra ser mais eficaz". A hipótese mais plausivel para isso se trata do fato de que nossa amostra é composta por profissionais jovens, com pouco tempo de formação acadêmica e com certo predomínio de pós-graduação em pediatria, o que sugere constante aperfeiçoamento técnico-científico. De qualquer forma, ainda são necessários ensaios clínicos controlados e randomizados que possam averiguar a efetividade de cada intervenção fisioterapêutica, objetivando investigar se existe superioridade entre elas.

O preenchimento do questionário por profissionais de um único estado da região Sul no Brasil se constitui como uma limitação deste estudo, dificultando a extrapolação dos dados 
em âmbito nacional. No entanto, este é o primeiro documento que se propôs a realizar um levantamento de intervenções para o público asmático infanto-juvenil. A ausência de conhecimento das regiões do estado e da origem dos hospitais destes profissionais também podem ser outras limitações dos resultados. Além disso, a variabilidade no aprendizado e na nomenclatura de algumas intervenções de FR pode ser outra limitação destes achados, visto que não existe um guia único recomendado para o entendimento desses manejos terapêuticos.

Em conclusão, os achados deste estudo evidenciaram, em uma amostra de fisioterapeutas do Sul do país, que as intervenções manuais (AFE, TEMP e ELPr), as técnicas de expectoração (tosse. huffing) e a aspiração de VAS visando à desobstrução brônquica são as mais frequentemente utilizadas no atendimento hospitalar de crianças e de adolescentes com asma. As intervenções se relacionaram com a faixa etária, considerando que houve menos relatos de intervenções independentes no grupo dos lactentes e menos aplicações terapeuta-dependentes nos escolares e adolescentes. Além disso, a utilização das técnicas de FR parece ter como base a prática clínica adquirida ao longo da experiência profissional, já que as evidências científicas quanto à efetividade das intervenções ainda são incipientes.

\section{Notas}

\section{Apoio financeiro}

Este estudo não recebeu apoio financeiro de fontes externas.

\section{Declaração de conflito de interesses}

Os autores declaram não haver conflitos de interesses relevantes ao conteúdo deste estudo.

\section{Contribuições dos autores}

Todos os autores fizeram contribuições substanciais para concepção, ou delineamento, ou aquisição, ou análise ou interpretação de dados; e redação do trabalho ou revisão crítica; e aprovação final da versão para publicação.

\section{Disponibilidade dos dados e responsabilidade pelos resultados}

Todos os autores declaram ter tido total acesso aos dados obtidos e assumem completa responsabilidade pela integridade destes resultados.

\section{Referências}

1. Macêdo TM, Freitas DA, Chaves GS, Holloway EA Mendonça KM. Breathing exercises for children with asthma. Cochrane Database Syst Rev. 2016;4(4):CD011017. https://doi.org/10.1002/14651858.CD011017.pub2

2. Roncada C, Oliveira SG, Cidade SF, Sarria EE, Mattiello $\mathrm{R}$, Ojeda BS, et al. Burden of asthma among inner-city children from Southern Brazil. J Asthma. 2016:53(5):498504. https://doi.org/10.3109/02770903.2015.1108438

3. Dias CS, Dias MAS, Friche AAL, Almeida MCM, Viana TC, Mingoti AS. Temporal and spatial trends in childhood asthma-related hospitalizations in Belo Horizonte, Minas Gerais, Brazil and their association with social vulnerability. Int J Environ Res Public Health. 2016;13(7):1-13. https://doi.org/10.3390/ijerph13070704

4. Solé D, Nunes, I, Wandalsen GF, Mallozi MC. Asthma in children and adolescents in Brazil: contribution of the International Study of Asthma and Allergies in Childhood (ISAAC). Rev Paul Pediatr. 2014:32(1):114-25. https://doi.org/10.1590/S0103-05822014000100018

5. Cardoso TA, Roncada C, Silva ER, Pinto LA, Jones $\mathrm{MH}$, Stein RT, et al. The impact of asthma in Brazil: a longitudinal analysis of data from a Brazilian national database system. J Bras Pneumol. 2017;43(3):163-68. https://doi.org/10.1590/s1806-37562016000000352

6. Amaral LM, Palma PV, Leite ICG. Asthma from the perspective of public health: epidemiology, economic impact and public policy. Rev APS. 2012;15(4):508-16.

7. IV Diretrizes Brasileiras para o Manejo da Asma. J Bras Pneumol. 2006:32 Suppl 7:447-74. https://doi. org/10.1590/S1806-37132006001100002

8. Tecklin JS. Physical therapy for children with chronic lung disease. Phys Ther. 1981; 61(12):1774-82. https:// doi.org/10.1093/ptj/61.12.1774

9. Zhang W, Wang Q, Liu L, Yang W, Liu H. Effects of physical therapy on lung function in children with asthma: a systematic review and meta-analysis. Pediatr Res. 2021;89:134356. https://doi.org/10.1038/s41390-020-0874-X

10. Silva PS, Barreto SS. Noninvasive ventilation in status asthmaticus in children: levels of evidence. Rev Bras Ter Intensiva. 2015;27(4):390-96. http://dx.doi. org/10.5935/0103-507X.20150065

11. Laurino RA, Barnabé V, Saraiva-Romanholo BM, Stelmach R, Cukier A, Nunes Mdo P. Respiratory rehabilitation: a physiotherapy approach to the control of asthma symptoms and anxiety. Clinics (Sao Paulo). 2012;67(11):1291-7. https://doi.org/10.6061/clinics/2012(11)12 
12. Bruurs ML, Van der Giessen LJ, Moed H. The effectiveness of physiotherapy in patients with asthma: $A$ systematic review of the literature. Respir Med. 2013; 107(4):483-94. https://doi.org/10.1016/j.rmed.2012.12.017

13. Das RR, Sankar J, Kabra SK. Role of breathing exercises and yoga/pranayama in childhood asthma: a systematic review. Curr Pediatr Rev. 2019:15(3):175-83. https://doi.org/10.2174/1573396315666190121122452

14. Lanza FC, Corso SD. Fisioterapia no paciente com asma: intervenção baseada em evidências. Arq Asma Alerg Imunol. 2017:1(1):59-64. https://doi. org/10.5935/2526-5393.20170008

15. Asher MI, Douglas C, Airy M, Andrews D, Trenholme A. Effects of chest physical therapy on lung function in children recovering from acute severe asthma. Pediatr Pulmonol. 1990;9(3):146-51. https://doi.org/10.1002/ ppul.1950090305

16. Paulin E, Favoreto PB, Vidotto CC. Benefits of respiratory physiotherapy in asthma case report. Arq Ciênc Saúde Unipar. 2001:5(2):149-54. https://revistas.unipar. br/index.php/saude/article/view/1119/982

17. Herrera AM, Cavada CHG, Mañalich MJ. Pediatric asthma hospitalization in Chile: 2001-2014. Rev Chil Pediatr. 2017;88(5):602-07. http://dx.doi.org/10.4067/ S0370-41062017000500005

18. Donadio MVF, Campos NE, Vendrusculo FM, Stofella AM, Almeida ACDS, Ziegler B, et al. Respiratory physical therapy techniques recommended for patients with cystic fibrosis treated in specialized centers. Braz J Phys Ther. 2020;24(6):532-8. http://dx.doi.org/10.1016/j. bjpt.2019.11.003

19. Sociedade Brasileira de Pediatria. Manual de orientação para a alimentação do lactente, do pré-escolar, do escolar, do adolescente e na escola. Departamento de Nutrologia. 3 ed. Rio de Janeiro: Sociedade Brasileira de Pediatria; 2012. p. 17-53.

20. Andrade RF, Paixao A. Principais técnicas fisioterapêuticas utilizadas no tratamento da criança asmáticarevisão. Rev Pediatria SOPERJ [Internet]. 2006 [cited 2020 Out 10];7(1):4-9. Available from: http://revistadepediatriasoperj.org.br/detalhe_artigo.asp?id=54

21. Ribeiro MAGO. Técnicas de remoção de secreção das vias aéreas. ASSOBRAFIR Ciência. 2019;10(Supl1):6198. http://dx.doi.org/10.47066/2177-9333/ac.36631

22. Morrow BM. Airway clearance therapy in acute paediatric respiratory illness: A state-of-the-art review. S Afr J Physiother. 2019:75(1):a1295. http://dx.doi. org/10.4102/sajp.v75i1.12.95

23. Rodrigues MS, Galvão IM. Pathophysiological aspects of cough reflex: a literature. Rev Med (São Paulo). 2017:96(3):172-6. http://dx.doi.org/10.11606/issn.16799836.v96i3p172-176

24. Nogueira MCP, Ribeiro SNS, Silva EP, Guimarães CL, Wandalsen GF, Solé D, et al. Is prolonged slow expiration a reproducible airway clearance technique? Phys Ther. 2019; 99(9):1224-30. http://dx.doi.org/10.1093/ptj/pzz080
25. Antunes LCO, Silva EG, Bocardo P, Daher DR, Faggiotto RD, Rugolo LMSS. Efeitos da fisioterapia respiratória convencional versus aumento do fluxo expiratório na saturação de $\mathrm{O} 2$, freqüência cardiaca e freqüência respiratória, em prematuros no periodo pós-extubação. Rev Bras Fisioter. 2006;10(1):97-103. http://dx.doi. org/10.1590/S1413-35552006000100013

26. Lanza FC, Gazzotti MR, Augusto LA, Mendes LMS, de Paula C, Solé D. Oscilação oral de alta frequência reduz a obstrução das vias aéreas em crianças com pneumonia? Rev Bras Alerg Imunopatol. 2009:32(2):59-62.

27. Veronezi J, Scortegagna D. Respiratory physiotherapy in cystic fibrosis. Rev HCPA. 2011;31(2):192-6.

28. Santana JCB, Barreto SSM, Carvalho PRA. Fatores associados com asma aguda grave na infância - aspectos epidemiológicos e clínicos. J Pediatr (Rio J). 1997:73(5):324-34. http://dx.doi.org/10.2223/JPED.548

29. Muchão FP, Silva-Filho LVRF. Avanços na inaloterapia em pediatria. J. Pediatr. (Rio J.) 2010;86(5):367-76. https://doi.org/10.1590/S0021-75572010000500004

30. Roncada C, Andrade J, Bischoff LC, Pitrez PM. Comparação de duas técnicas inalatórias para administrar broncodilatador em crianças e adolescentes com crise aguda de asma: metanálise. Rev Paul Pediatr. 2018; 36(3):364-71. https://doi.org/10.1590/19840462/:2018:36:3:00002

31. Matilde INE, Eid RAC, Nunes AF, Ambrozin ARP, Moura RH, Carnieli-Cazati, et al. Bronchial hygiene techniques in patients on mechanical ventilation: what are used and why? Einstein (Sao Paulo). 2018;16(1):1-7. https://doi.org/10.1590/S1679-45082018AO3856

\section{Daniele Oppermann Ruckert}

Bacharel em Fisioterapia pelo Centro Universitário Cenecista de Osório (UNICNEC), em Osório, RS, Brasil.

\section{Márcio Vinícius Fagundes Donadio}

Doutor em Ciências Biológicas (Fisiologia) pela Universidade Federal do Rio Grande do Sul (UFRGS), em Porto Alegre, RS, Brasil; professor da Pontifícia Universidade Católica do Rio Grande do Sul (PUCRS), em Porto Alegre, RS, Brasil.

\section{João Paulo Heinzmann-Filho}

Doutor em Saúde da Criança pela Pontifícia Universidade Católica do Rio Grande do Sul (PUCRS), em Porto Alegre, RS, Brasil; professor do Centro Universitário Cenecista de Osório (UNICNEC), em Osório, RS, Brasil. 


\section{Endereço para correspondência}

João Paulo Heinzmann-Filho

Centro Universitário Cenecista de Osório

Rua Vinte e Quatro de Maio, 141

Centro, 95520-000

Osório, RS, Brasil

Os textos deste artigo foram revisados pela Poá Comunicação e submetidos para validação do(s) autor(es) antes da publicação. 